\title{
DIAGONALITY OF ACTIONS AND KMS WEIGHTS
}

\author{
JOHANNES CHRISTENSEN AND KLAUS THOMSEN
}

\section{INTRODUCTION}

Recent years have seen an increasing interest in the investigation of KMS states for one-parameter actions on $C^{*}$-algebras. While the original motivation for the introduction of KMS states came from the interpretation of these states as equilibrium states in models from quantum statistical mechanics, the renewed interest stems also from more purely mathematical considerations, where the KMS states have been related to objects and structures from other fields, such as number theory or dynamical systems. In the present paper we investigate relations between properties of the KMS states and properties of the one-parameter action giving rise to them. As we shall now explain, we show that the existence of a 'diagonal' KMS state or weight implies that the action itself must be 'diagonal'.

For most if not all the one-parameter actions on $C^{*}$-algebras for which we have been able to determine the structure of the KMS states or KMS weights, the underlying $C^{*}$-algebra can be presented as the $C^{*}$-algebra of a locally compact groupoid, as introduced by Renault in [Re1], and the action described as arising from a continuous real-valued homomorphism on the groupoid by a canonical procedure also introduced in [Re1]. For this reason the results of Neshveyev, [N], which extend results of Renault and give a general and abstract description of the KMS states for such actions on a groupoid $C^{*}$-algebra is of outmost importance. In the following we call these actions diagonal.

When the groupoid and the associated $C^{*}$-algebra is fixed, it is certainly not all one-parameter actions that are diagonal. It follows from Neshveyev's theorem, Theorem 1.3 in [N], that a diagonal action has the property that if a KMS state exists, there will also be one which factorises through the canonical conditional expectation onto the abelian $C^{*}$-subalgebra generated by the continuous compactly supported functions on the unit space. In the following we call these states diagonal. The present work sprang from the realisation that in many cases the property that there is a diagonal KMS state, characterises the diagonal actions. That is, for many groupoid $C^{*}$-algebras a one-parameter action is diagonal if and only if the action admits a diagonal KMS state. The simplest example of this is perhaps the following.

Consider the $C^{*}$-algebra $M_{n}$ of complex $n$ by $n$ matrices. A continuous oneparameter group $\alpha$ of automorphisms on $M_{n}$ is inner in the sense that there is a self-adjoint matrix $A \in M_{n}$ such that

$$
\alpha_{t}(B)=e^{i t A} B e^{-i t A}
$$

Version: March 5, 2022. 
for all $t \in \mathbb{R}$ and all $B \in M_{n}$. For each $\beta \in \mathbb{R}$ there is a unique $\beta$-KMS state $\omega_{\beta}$ for $\alpha$ given by

$$
\omega_{\beta}(B)=\frac{\operatorname{Tr}\left(e^{-\beta A} B\right)}{\operatorname{Tr}\left(e^{-\beta A}\right)} .
$$

It can be shown that for $\beta \neq 0$ the state $\omega_{\beta}$ factorises through the canonical (and unique) conditional expectation from $M_{n}$ onto the $C^{*}$-subalgebra of diagonal matrices if and only if $A$ is diagonal. It is this fact we will generalize. For this note that $M_{n}$ is the groupoid $C^{*}$-algebra of the groupoid $\mathcal{G}=\{1,2,3, \cdots, n\} \times\{1,2,3, \cdots, n\}$ with operations

$$
(a, b)(b, c)=(a, c) \text { and }(a, b)^{-1}=(b, a) .
$$

When $M_{n}$ is identified with the $C^{*}$-algebra $C^{*}(\mathcal{G})$ of $\mathcal{G}$, the diagonal matrices in $M_{n}$ constitute the $C^{*}$-algebra $C\left(\mathcal{G}^{(0)}\right)$ of (continuous) functions on $\mathcal{G}$ whose support is contained in the unit space

$$
\mathcal{G}^{(0)}=\{(k, k): k \in\{1,2, \cdots, n\}\}
$$

of $\mathcal{G}$. In this picture the conditional expectation onto the diagonal matrices is the map

$$
P: C^{*}(\mathcal{G}) \rightarrow C\left(\mathcal{G}^{(0)}\right)
$$

which restricts functions to $\mathcal{G}^{(0)}$. Furthermore, the matrix $A$ will be diagonal if and only if there is a groupoid homomorphism $c: \mathcal{G} \rightarrow \mathbb{R}$ such that

$$
\alpha_{t}(f)(a, b)=e^{i c(a, b) t} f(a, b)
$$

for all $t \in \mathbb{R},(a, b) \in \mathcal{G}$ and all $f \in C^{*}(\mathcal{G})$. Because the whole setup is so transparent in this case, we can easily conclude that there is an equivalence between the following conditions:

1) $\alpha$ is diagonal in the sense that there is a groupoid homomorphism $c: \mathcal{G} \rightarrow \mathbb{R}$ such that (1.1) holds.

2) There is a $\beta \neq 0$ and a $\beta$-KMS state $\omega_{\beta}$ for $\alpha$ which is diagonal in the sense that it factorises through the conditional expectation $C^{*}(\mathcal{G}) \rightarrow C\left(\mathcal{G}^{(0)}\right)$.

3) $\alpha_{t}(f)=f$ for all $t \in \mathbb{R}$ and all $f \in C\left(\mathcal{G}^{(0)}\right)$.

4) All $\beta$-KMS states of $\alpha$, for $\beta \neq 0$, are diagonal.

Our main result is that these equivalences hold much more generally as we shall now explain.

\section{Notation AND MAIN RESUlT}

Let $\mathcal{G}$ be a second countable locally compact Hausdorff étale groupoid with unit space $\mathcal{G}^{(0)}$. Let $r: \mathcal{G} \rightarrow \mathcal{G}^{(0)}$ and $s: \mathcal{G} \rightarrow \mathcal{G}^{(0)}$ be the range and source maps, respectively. For $x \in \mathcal{G}^{(0)}$ put $\mathcal{G}^{x}=r^{-1}(x), \mathcal{G}_{x}=s^{-1}(x)$ and $\mathcal{G}_{x}^{x}=s^{-1}(x) \cap r^{-1}(x)$. Note that $\mathcal{G}_{x}^{x}$ is a group, the isotropy group at $x$. The space $C_{c}(\mathcal{G})$ of continuous compactly supported functions is a $*$-algebra when the product is defined by

$$
\left(f_{1} * f_{2}\right)(g)=\sum_{h \in \mathcal{G}^{r(g)}} f_{1}(h) f_{2}\left(h^{-1} g\right)
$$

and the involution by $f^{*}(g)=\overline{f\left(g^{-1}\right)}$. To define the reduced groupoid $C^{*}$-algebra $C_{r}^{*}(\mathcal{G})$, let $x \in \mathcal{G}^{(0)}$. There is a representation $\pi_{x}$ of $C_{c}(\mathcal{G})$ on the Hilbert space $l^{2}\left(\mathcal{G}_{x}\right)$ 
of square-summable functions on $\mathcal{G}_{x}$ given by

$$
\pi_{x}(f) \psi(g)=\sum_{h \in \mathcal{G}^{r(g)}} f(h) \psi\left(h^{-1} g\right) .
$$

$C_{r}^{*}(\mathcal{G})$ is the completion of $C_{c}(\mathcal{G})$ with respect to the norm

$$
\|f\|_{r}=\sup _{x \in \mathcal{G}^{(0)}}\left\|\pi_{x}(f)\right\| .
$$

Note that $C_{r}^{*}(\mathcal{G})$ is separable since we assume that the topology of $\mathcal{G}$ is second countable.

We shall here be concerned not only with KMS states, but more generally with KMS weights. Let $A$ be a $C^{*}$-algebra and $A_{+}$the convex cone of positive elements in $A$. A weight on $A$ is a map $\psi: A_{+} \rightarrow[0, \infty]$ with the properties that $\psi(a+b)=$ $\psi(a)+\psi(b)$ and $\psi(\lambda a)=\lambda \psi(a)$ for all $a, b \in A_{+}$and all $\lambda \in \mathbb{R}, \lambda>0$. By definition $\psi$ is densely defined when $\left\{a \in A_{+}: \psi(a)<\infty\right\}$ is dense in $A_{+}$and lower semicontinuous when $\left\{a \in A_{+}: \psi(a) \leq \alpha\right\}$ is closed for all $\alpha \geq 0$. We will use [Ku], [KV] as our source for information on weights, and we say that a weight is proper when it is non-zero, densely defined and lower semi-continuous. Let $\psi$ be a proper weight on $A$. Set $\mathcal{N}_{\psi}=\left\{a \in A: \psi\left(a^{*} a\right)<\infty\right\}$ and note that

$$
\mathcal{M}_{\psi}=\operatorname{Span}\left\{a^{*} b: a, b \in \mathcal{N}_{\psi}\right\}
$$

is a dense *-subalgebra of $A$, and that there is a unique well-defined linear map $\mathcal{M}_{\psi} \rightarrow \mathbb{C}$ which extends $\psi: \mathcal{M}_{\psi} \cap A_{+} \rightarrow[0, \infty)$. We denote also this densely defined linear map by $\psi$.

Let $\alpha: \mathbb{R} \rightarrow$ Aut $A$ be a continuous one-parameter group of automorphisms on $A$. Let $\beta \in \mathbb{R}$. Following [C] we say that a proper weight $\psi$ on $A$ is a $\beta$-KMS weight for $\alpha$ when

i) $\psi \circ \alpha_{t}=\psi$ for all $t \in \mathbb{R}$, and

ii) for every pair $a, b \in \mathcal{N}_{\psi} \cap \mathcal{N}_{\psi}^{*}$ there is a continuous and bounded function $F$ defined on the closed strip $D_{\beta}$ in $\mathbb{C}$ consisting of the numbers $z \in \mathbb{C}$ whose imaginary part lies between 0 and $\beta$, and is holomorphic in the interior of the strip and satisfies that

$$
F(t)=\psi\left(a \alpha_{t}(b)\right), F(t+i \beta)=\psi\left(\alpha_{t}(b) a\right)
$$

for all $t \in \mathbb{R}$.

Compared to $[\mathrm{C}]$ we have changed the orientation in order to have the same sign convention as in $[\mathrm{BR}]$, for example. It will be important for us that there is an alternative characterisation of when a proper weight is a $\beta$-KMS weight. Specifically, by Proposition 1.11 in [KV] a proper weight $\psi$ is a $\beta$-KMS weight for $\alpha$ if and only if it is $\alpha$-invariant (as in i) above) and

$$
\psi\left(a^{*} a\right)=\psi\left(\alpha_{-\frac{i \beta}{2}}(a) \alpha_{-\frac{i \beta}{2}}(a)^{*}\right)
$$

for all $a$ in the domain $D\left(\alpha_{-\frac{i \beta}{2}}\right)$ of $\alpha_{-\frac{i \beta}{2}}$; the closure of the restriction of $\alpha_{-\frac{i \beta}{2}}$ to the analytic elements for $\alpha$, cf. $[\mathrm{Ku}]$. A $\beta$-KMS weight $\psi$ with the property that

$$
\sup \{\psi(a): 0 \leq a \leq 1\}=1
$$

will be called a $\beta-K M S$ state. 
Returning to the case $A=C_{r}^{*}(\mathcal{G})$, note that the map $C_{c}(\mathcal{G}) \rightarrow C_{c}\left(\mathcal{G}^{(0)}\right)$ which restricts functions to $\mathcal{G}^{(0)}$ extends to a conditional expectation $P: C_{r}^{*}(\mathcal{G}) \rightarrow C_{0}\left(\mathcal{G}^{(0)}\right)$. Via $P$ a regular Borel measure $m$ on $\mathcal{G}^{(0)}$ gives rise to a weight $\varphi_{m}: C_{r}^{*}(\mathcal{G})_{+} \rightarrow[0, \infty]$ defined by the formula

$$
\varphi_{m}(a)=\int_{\mathcal{G}^{(0)}} P(a) d m
$$

It follows from Fatou's lemma that $\varphi_{m}$ is lower semi-continuous. Since $\varphi_{m}(f a f)<\infty$ for every non-negative function $f$ in $C_{c}\left(\mathcal{G}^{(0)}\right)$, it follows that $\varphi_{m}$ is also densely defined, i.e. $\varphi_{m}$ is a proper weight on $C_{r}^{*}(\mathcal{G})$ if and only if $m$ is not the zero measure. In the following we say that a proper weight $\psi$ on $C_{r}^{*}(\mathcal{G})$ is diagonal when $\psi=\varphi_{m}$ for some regular Borel measure $m$ on $\mathcal{G}^{(0)}$. By the Riesz representation theorem this occurs if and only if $\psi \circ P=\psi$.

Given a continuous homomorphism $c: \mathcal{G} \rightarrow \mathbb{R}$ there is a continuous one-parameter group $\sigma^{c}$ on $C_{r}^{*}(\mathcal{G})$ such that

$$
\sigma_{t}^{c}(g)(\xi)=e^{i t c(\xi)} g(\xi)
$$

for all $t \in \mathbb{R}$, all $g \in C_{c}(\mathcal{G})$ and all $\xi \in \mathcal{G}$, cf. [Re1]. A one-parameter action of this kind will be called diagonal in the following. We can then formulate our main result as follows.

Theorem 2.1. Let $\mathcal{G}$ be a locally compact second countable Hausdorff étale groupoid such that for at least one element $x \in \mathcal{G}^{(0)}$ the isotropy group $\mathcal{G}_{x}^{x}$ is trivial, i.e. $\mathcal{G}_{x}^{x}=\{x\}$, and that $\mathcal{G}$ is minimal in the sense that $s\left(r^{-1}(y)\right)$ is dense in $\mathcal{G}^{(0)}$ for all $y \in \mathcal{G}^{(0)}$. Furthermore, assume that $\mathcal{G}^{(0)}$ is totally disconnected.

Let $\alpha=\left(\alpha_{t}\right)_{t \in \mathbb{R}}$ be a continuous one-parameter group of automorphisms on $C_{r}^{*}(\mathcal{G})$ and assume that for some $\beta_{0} \neq 0$ there is a $\beta_{0}-K M S$ weight for $\alpha$.

The following are equivalent:

1) There is a $\beta_{1} \neq 0$ and a diagonal $\beta_{1}-K M S$ weight for $\alpha$.

2) Whenever $\beta \neq 0$ and there is a $\beta$-KMS weight for $\alpha$, there is also a diagonal $\beta$-KMS weight for $\alpha$.

3) $\alpha_{t}(f)=f$ for all $t \in \mathbb{R}$ and all $f \in C_{0}\left(\mathcal{G}^{(0)}\right)$.

4) $\alpha_{t}\left(C_{0}\left(\mathcal{G}^{(0)}\right)\right) \subseteq C_{0}\left(\mathcal{G}^{(0)}\right)$ for all $t \in \mathbb{R}$.

5) $\alpha$ is diagonal.

Some of the (non-trivial) implications hold with fewer assumptions. Specifically, $1) \Rightarrow 3$ ) holds without the assumption that the unit space is totally disconnected by Proposition 4.1 , and the implication 3$) \Rightarrow 5$ ) holds assuming only that the points with trivial isotropy are dense in $\mathcal{G}^{(0)}$ (i.e. if $\mathcal{G}$ is topologically principal) by Proposition 4.4, The implication 5) $\Rightarrow 2$ ) holds whenever $\mathcal{G}^{(0)}$ is totally disconnected, without any further assumptions, as it follows from Corollary 3.4. It may be that this implication is true in general and if so the theorem with 4) removed is true also when $\mathcal{G}^{(0)}$ is not totally disconnected. However, the first two assumptions on $\mathcal{G}$ which are equivalent to topological principality and minimality of $\mathcal{G}$ are certainly necessary for the implication 3) $\Rightarrow 1$ ) to hold, cf. Example 4.10. Finally, the gauge action on the $C^{*}$-algebra of a strongly connected (row-finite) graph with infinite Gurevich entropy does not admit any KMS weights at all, cf. [Th3], showing that it is necessary to assume the existence of some KMS-weight for the implication 5) $\Rightarrow 1$ ) to hold. 


\section{NeShVEYEV'S THEOREM FOR KMS WEIGHTS}

Lemma 3.1. Let $A$ be a $C^{*}$-algebra, $\alpha$ a continuous one-parameter group of automorphisms on $A$ and $\psi$ a KMS weight for $\alpha$. Let $p \in A$ be a projection in the fixed point algebra of $\alpha$. Then $\psi(p)<\infty$.

Proof. Assume that $a \geq 0, \psi(a)<\infty$ and that $a^{\frac{1}{2}}$ is analytic for $\alpha$. Then Proposition 1.11 in $[\mathrm{KV}]$ applies to conclude that

$$
\psi(\text { pap })=\psi\left(\alpha_{-\frac{i \beta}{2}}\left(a^{\frac{1}{2}}\right) p \alpha_{-\frac{i \beta}{2}}\left(a^{\frac{1}{2}}\right)^{*}\right) \leq \psi\left(\alpha_{-\frac{i \beta}{2}}\left(a^{\frac{1}{2}}\right) \alpha_{-\frac{i \beta}{2}}\left(a^{\frac{1}{2}}\right)^{*}\right)=\psi(a) .
$$

Let $\left\{b_{k}\right\}$ be a sequence of positive elements in $A$ such that $\lim _{k \rightarrow \infty} b_{k}=p$ and $\psi\left(b_{k}\right)<\infty$ for all $k$. For each $n \in \mathbb{N}$, set

$$
c_{k, n}=\sqrt{\frac{n}{\pi}} \int_{\mathbb{R}} \alpha_{t}\left(b_{k}\right) e^{-n t^{2}} d t .
$$

Then $c_{k, n}$ is analytic for $\alpha$ and $\psi\left(c_{k, n}^{2}\right) \leq\left\|c_{k, n}\right\| \psi\left(c_{k, n}\right) \leq\left\|c_{k, n}\right\| \psi\left(b_{k}\right)<\infty$ for all $k, n$. It follows therefore from (3.1) that $\psi\left(p c_{k, n}^{2} p\right) \leq \psi\left(c_{k, n}^{2}\right)<\infty$ for all $k, n$. Note that

$$
\lim _{k \rightarrow \infty} \lim _{n \rightarrow \infty} c_{k, n}^{2}=\lim _{k \rightarrow \infty} b_{k}^{2}=p^{2}=p .
$$

It follows that there are $k, n$ such that $\left\|p-p c_{k, n}^{2} p\right\| \leq \frac{1}{2}$, and then spectral theory tells us that $p c_{k, n}^{2} p \geq \frac{1}{2} p$. Hence $\psi(p) \leq 2 \psi\left(p c_{k, n}^{2} p\right)<\infty$.

Let $\mathcal{G}$ be a locally compact second countable Hausdorff étale groupoid and $c$ : $\mathcal{G} \rightarrow \mathbb{R}$ a continuous homomorphism. Let $\mu$ be a regular Borel measure on $\mathcal{G}^{(0)}$ and $\beta \in \mathbb{R}$ a real number. We say that $\mu$ is $(\mathcal{G}, c)$-conformal with exponent $\beta$, as in [Th3], or that $\mu$ is quasi-invariant with Radon-Nikodym cocycle $e^{-\beta c}$, as in [N], when

$$
\mu(s(W))=\int_{r(W)} e^{\beta c\left(r_{W}^{-1}(x)\right)} d \mu(x)
$$

for every open bi-section $W \subseteq \mathcal{G}$, where $r_{W}^{-1}$ denotes the inverse $r: W \rightarrow r(W)$. For each $x \in \mathcal{G}^{(0)}$ we can consider the full group $C^{*}$-algebra $C^{*}\left(\mathcal{G}_{x}^{x}\right)$ of the discrete group $\mathcal{G}_{x}^{x}$, the isotropy group at $x$. As in [N] we denote for $g \in \mathcal{G}_{x}^{x}$ by $u_{g}$ the characteristic function of the element $g$ when we consider $C^{*}\left(\mathcal{G}_{x}^{x}\right)$ as a completion of $C_{c}\left(\mathcal{G}_{x}^{x}\right)$. Thus $u_{g}, g \in \mathcal{G}_{x}^{x}$, are the canonical unitary generators of $C^{*}\left(\mathcal{G}_{x}^{x}\right)$. Following [N] we say that a collection $\varphi_{x}, x \in \mathcal{G}^{(0)}$, of states on $C^{*}\left(\mathcal{G}_{x}^{x}\right)$ is a $\mu$-measurable field when the function

$$
\mathcal{G}^{(0)} \ni x \mapsto \sum_{g \in \mathcal{G}_{x}^{x}} f(g) \varphi_{x}\left(u_{g}\right)
$$

is $\mu$-measurable for all $f \in C_{c}(\mathcal{G})$. We identify two $\mu$-measurable fields $\left\{\varphi_{x}\right\}_{x \in \mathcal{G}^{(0)}}$ and $\left\{\varphi_{x}^{\prime}\right\}_{x \in \mathcal{G}^{(0)}}$ when $\varphi_{x}=\varphi_{x}^{\prime}$ for $\mu$-almost every $x$.

The following theorem is a version for weights of Theorem 1.3 in [N]. Note that it deals with the full groupoid $C^{*}$-algebra $C^{*}(\mathcal{G})$ which is an extension of the reduced groupoid $C_{r}^{*}(\mathcal{G})$. We refer to [Re1] for the definition of the full groupoid $C^{*}$-algebra. To understand the following theorem and its proof it suffices to know that $C^{*}(\mathcal{G})$, like $C_{r}^{*}(\mathcal{G})$, is a completion of $C_{c}(\mathcal{G})$ and that a continuous homomorphism $c: \mathcal{G} \rightarrow \mathbb{R}$ also defines a continuous one-parameter group $\sigma^{c}$ on $C^{*}(\mathcal{G})$ via the formula (2.3). 
Theorem 3.2. (Neshveyev's theorem for weights.) Let $\mathcal{G}$ be a locally compact second countable Hausdorff étale groupoid and let $c: \mathcal{G} \rightarrow \mathbb{R}$ be a continuous homomorphism. Assume that the unit space $\mathcal{G}^{(0)}$ of $\mathcal{G}$ is totally disconnected.

There is a bijective correspondence between the $\beta$-KMS weights for $\sigma^{c}$ on $C^{*}(\mathcal{G})$ and the pairs $\left(\mu,\left\{\varphi_{x}\right\}_{x \in \mathcal{G}^{(0)}}\right)$, where $\mu$ is a regular Borel measure on $\mathcal{G}^{(0)}$ and $\left\{\varphi_{x}\right\}_{x \in \mathcal{G}^{(0)}}$ is a $\mu$-measurable field of states $\varphi_{x}$ on $C^{*}\left(\mathcal{G}_{x}^{x}\right)$ such that

a) $\mu$ is quasi-invariant with Radon-Nikodym cocycle $e^{-\beta c}$,

b) $\varphi_{x}\left(u_{g}\right)=\varphi_{r(h)}\left(u_{h g h^{-1}}\right)$ for $\mu$-almost every $x \in \mathcal{G}^{(0)}$ and all $g \in \mathcal{G}_{x}^{x}, h \in \mathcal{G}_{x}$, and

c) $\varphi_{x}\left(u_{g}\right)=0$ for $\mu$-almost every $x \in \mathcal{G}^{(0)}$ and all $g \in \mathcal{G}_{x}^{x} \backslash c^{-1}(0)$.

The $\beta$-KMS weight $\phi$ corresponding to the pair $\left(\mu,\left\{\varphi_{x}\right\}_{x \in \mathcal{G}^{(0)}}\right)$ has the properties that $C_{c}(\mathcal{G}) \subseteq \mathcal{M}_{\phi}$ and

$$
\phi(f)=\int_{\mathcal{G}^{(0)}} \sum_{g \in \mathcal{G}_{x}^{x}} f(g) \varphi_{x}\left(u_{g}\right) d \mu(x)
$$

when $f \in C_{c}(\mathcal{G})$.

Proof. Let $\phi$ be a $\beta$-KMS weight for $\sigma^{c}$. Since $\mathcal{G}^{(0)}$ is totally disconnected by assumption there is a sequence $p_{1} \leq p_{2} \leq p_{3} \leq \cdots$ of projections in $C_{c}\left(\mathcal{G}^{(0)}\right)$ with the property that $\left\{p_{n}\right\}$ is an approximate unit for $C^{*}(\mathcal{G})$. It follows from Lemma 3.1 that $\phi\left(p_{n}\right)<\infty$ for all $n$. Since $\phi \neq 0$ we can assume, without loss of generality, that $\phi\left(p_{n}\right)>0$ for all $n$. Since $\phi(f)<\infty$ for every non-negative function in $C_{c}\left(\mathcal{G}^{(0)}\right)$ it follows that $C_{c}(\mathcal{G}) \subseteq \mathcal{M}_{\phi}$ and from the Riesz representation theorem that there is a unique regular Borel measure $\mu$ on $\mathcal{G}^{(0)}$ such that

$$
\phi(f)=\int_{\mathcal{G}^{(0)}} f(x) d \mu(x)
$$

for all $f \in C_{c}\left(\mathcal{G}^{(0)}\right)$. Let $U_{n}$ be the compact and open support of $p_{n}$, and set

$$
\mathcal{G}^{n}=\left.\mathcal{G}\right|_{U_{n}}=\left\{\xi \in \mathcal{G}: r(\xi), s(\xi) \in U_{n}\right\}
$$

and $c_{n}=\left.c\right|_{\mathcal{G}^{n}}$. Note that $\phi\left(p_{n}\right)^{-1} \phi$ restricts to a $\beta$-KMS state on $p_{n} C^{*}(\mathcal{G}) p_{n}=$ $C^{*}\left(\mathcal{G}^{n}\right)$. It follows from Neshveyev's theorem [N] that there is a probability measure $\mu_{n}$ on $U_{n}$, and a $\mu_{n}$-measurable field $\left\{\varphi_{x}^{n}\right\}_{x \in U_{n}}$ of states such that

an) $\mu_{n}$ is quasi-invariant on $\mathcal{G}^{n}$ with cocycle $e^{-\beta c_{n}}$.

bn) $\varphi_{x}^{n}\left(u_{g}\right)=\varphi_{r(h)}^{n}\left(u_{h g h^{-1}}\right)$ for $\mu_{n^{-}}$-almost every $x \in U_{n}$ and all $g \in \mathcal{G}_{x}^{x}, h \in\left(\mathcal{G}^{n}\right)_{x}$,

cn) $\varphi_{x}^{n}\left(u_{g}\right)=0$ for $\mu_{n}$-almost every $x \in U_{n}$ and all $g \in \mathcal{G}_{x}^{x} \backslash c_{n}^{-1}(0)$,

and

$$
\phi\left(p_{n}\right)^{-1} \phi(f)=\int_{U_{n}} \sum_{g \in \mathcal{G}_{x}^{x}} f(g) \varphi_{x}^{n}\left(u_{g}\right) d \mu_{n}(x)
$$

when $f \in C_{c}\left(\mathcal{G}^{n}\right)$. For every $f \in C_{c}\left(U_{n}\right)$ we get that:

$$
\begin{aligned}
& \phi\left(p_{n}\right)^{-1} \int_{U_{n}} f(x) d \mu(x)=\phi\left(p_{n}\right)^{-1} \int_{\mathcal{G}^{(0)}} f(x) d \mu(x)=\phi\left(p_{n}\right)^{-1} \phi(f) \\
& =\int_{U_{n}} \sum_{g \in \mathcal{G}_{x}^{x}} f(g) \varphi_{x}^{n}\left(u_{g}\right) d \mu_{n}(x)=\int_{U_{n}} f(x) d \mu_{n}(x)
\end{aligned}
$$


so $\left.\mu\right|_{U_{n}}=\phi\left(p_{n}\right) \mu_{n}$. Notice that since $\phi\left(p_{n}\right)>0$, being a $\mu$ nullset in $U_{n}$ is the same as being a $\mu_{n}$ nullset. For a Borel set $V \subseteq U_{n} \subseteq U_{n+1}$ we have that:

$$
\phi\left(p_{n+1}\right) \mu_{n+1}(V)=\mu(V)=\phi\left(p_{n}\right) \mu_{n}(V)
$$

So $\mu_{n}=\phi\left(p_{n+1}\right) /\left.\phi\left(p_{n}\right) \mu_{n+1}\right|_{U_{n}}$. For every $f \in C_{c}\left(\mathcal{G}^{n}\right)$ we get that:

$$
\begin{aligned}
& \int_{U_{n}} \sum_{g \in \mathcal{G}_{x}^{x}} f(g) \varphi_{x}^{n}\left(u_{g}\right) d \mu_{n}(x)=\phi\left(p_{n}\right)^{-1} \phi(f)=\frac{\phi\left(p_{n+1}\right)}{\phi\left(p_{n}\right)} \phi\left(p_{n+1}\right)^{-1} \phi(f) \\
& =\frac{\phi\left(p_{n+1}\right)}{\phi\left(p_{n}\right)} \int_{U_{n+1}} \sum_{g \in \mathcal{G}_{x}^{x}} f(g) \varphi_{x}^{n+1}\left(u_{g}\right) d \mu_{n+1}(x)=\int_{U_{n}} \sum_{g \in \mathcal{G}_{x}^{x}} f(g) \varphi_{x}^{n+1}\left(u_{g}\right) d \mu_{n}(x)
\end{aligned}
$$

Since $\mu_{n}$ by choice satisfy an), and since it's easily seen that $\left\{\varphi_{x}^{n+1}\right\}_{x \in U_{n}}$ satisfy bn) and $\mathrm{cn}$ ), the uniqueness statement in Neshveyev's theorem gives that $\varphi_{x}^{n}=\varphi_{x}^{n+1}$ for a.e. $x \in U_{n}$. Hence for a.e. $x \in \mathcal{G}^{(0)}$ we can define a state on $C^{*}\left(\mathcal{G}_{x}^{x}\right)$ by:

$$
\varphi_{x}(d)=\lim _{n \rightarrow \infty} \varphi_{x}^{n}(d) .
$$

For every $f \in C_{c}(\mathcal{G})$ there is a $N \in \mathbb{N}$ such that $f \in C_{c}\left(\mathcal{G}^{N}\right)$, and hence:

$$
\phi(f)=\phi\left(P_{N}\right) \int_{U_{N}} \sum_{g \in \mathcal{G}_{x}^{x}} f(g) \varphi_{x}^{N}\left(u_{g}\right) d \mu_{N}(x)=\int_{\mathcal{G}^{(0)}} \sum_{g \in \mathcal{G}_{x}^{x}} f(g) \varphi_{x}\left(u_{g}\right) d \mu(x) .
$$

The properties a)-c) follow from an)-cn), and measurability of $x \mapsto \sum_{g \in \mathcal{G}_{x}^{x}} f(g) \varphi_{x}\left(u_{g}\right)$ follows from measurability of $x \mapsto \sum_{g \in \mathcal{G}_{x}^{x}} f(g) \varphi_{x}^{n}\left(u_{g}\right)$.

For the converse, assume we are given a pair $\left(\mu,\left\{\varphi_{x}\right\}_{x \in \mathcal{G}^{(0)}}\right)$ for which a),b) and c) hold. As shown by Neshveyev in the proof of Theorem 1.1 in $[\mathbb{N}]$ every $x$ gives rise to a state $\psi_{x}$ on $C^{*}(\mathcal{G})$ such that

$$
\psi_{x}(f)=\sum_{g \in \mathcal{G}_{x}^{x}} f(g) \varphi_{x}\left(u_{g}\right)
$$

when $f \in C_{c}(\mathcal{G})$. Note that $x \rightarrow \sum_{g \in \mathcal{G}_{x}^{x}} f(g) \varphi_{x}\left(u_{g}\right)$ is $\mu$-measurable by assumption, and then $x \rightarrow \psi_{x}(a)$ is also for each $a \in C^{*}(\mathcal{G})$. For $a \geq 0$ we can therefore define

$$
\phi(a)=\int_{\mathcal{G}^{(0)}} \psi_{x}(a) d \mu(x) .
$$

$\phi$ is a lower semi-continuous weight by Fatous lemma and by regularity

$$
\phi\left(p_{n} a p_{n}\right)=\int_{U_{n}} \psi_{x}(a) d \mu(x) \leq\|a\| \mu\left(U_{n}\right)<\infty
$$

for all $n$, so it is also densely defined. Note that $C_{c}(\mathcal{G}) \subseteq \mathcal{M}_{\phi}$ and that (3.3) holds by construction. Since the pair $\left(\phi\left(p_{n}\right)^{-1} \mu,\left\{\varphi_{x}\right\}_{x \in U_{n}}\right)$ represents $\phi\left(p_{n}\right)^{-1} \phi$ in the sense of Theorem 1.1 in $[\mathrm{N}]$ it follows from Theorem 1.3 in [N] that $\phi$ is a bounded $\beta$-KMS weight on $p_{n} C^{*}(\mathcal{G}) p_{n}$. Since

$$
\psi_{x}\left(p_{n} a p_{n}\right)=\left\{\begin{array}{ll}
\psi_{x}(a), & x \in U_{n} \\
0, & x \notin U_{n}
\end{array},\right.
$$

we find that $\lim _{n \rightarrow \infty} \phi\left(p_{n} a p_{n}\right)=\lim _{n \rightarrow \infty} \int_{U_{n}} \psi_{x}(a) d \mu(x)=\phi(a)$ for all $a \geq 0$ in $C^{*}(\mathcal{G})$. Now note that for every $a$ in the domain of $\sigma_{\frac{-i \beta}{2}}^{c}$,

$$
\phi\left(p_{n} a^{*} a p_{n}\right)=\phi\left(\sigma_{\frac{-i \beta}{2}}^{c}\left(a p_{n}\right) \sigma_{\frac{-i \beta}{2}}^{c}\left(a p_{n}\right)^{*}\right)=\phi\left(\sigma_{\frac{-i \beta}{2}}^{c}(a) p_{n} \sigma_{\frac{-i \beta}{2}}^{c}(a)^{*}\right)
$$


since $\phi$ is a bounded $\beta$-KMS weight on $p_{n} C^{*}(\mathcal{G}) p_{n}$. Since

$$
\lim _{n \rightarrow \infty} \phi\left(\sigma_{\frac{-i \beta}{2}}^{c}(a) p_{n} \sigma_{\frac{-i \beta}{2}}^{c}(a)^{*}\right)=\phi\left(\sigma_{\frac{-i \beta}{2}}^{c}(a) \sigma_{\frac{-i \beta}{2}}^{c}(a)^{*}\right)
$$

by the lower semi-continuity of $\phi$, we conclude that $\phi\left(a^{*} a\right)=\phi\left(\sigma_{\frac{-i \beta}{2}}^{c}(a) \sigma_{\frac{-i \beta}{2}}^{c}(a)^{*}\right)$, showing that $\phi$ is indeed a $\beta$-KMS weight for $\sigma^{c}$.

If $\left(\mu,\left\{\varphi_{x}\right\}_{x \in \mathcal{G}^{(0)}}\right)$ and $\left(\mu^{\prime},\left\{\varphi_{x}^{\prime}\right\}_{x \in \mathcal{G}^{(0)}}\right)$ represent the same $\beta$-KMS weight it follows from the uniqueness part of the Riesz representation theorem that $\mu=\mu^{\prime}$. By using (3.3) we find that

$$
\int_{\mathcal{G}^{(0)}} k(x) \sum_{g \in \mathcal{G}_{x}^{x}} f(g) \varphi_{x}\left(u_{g}\right) d \mu(x)=\int_{\mathcal{G}^{(0)}} k(x) \sum_{g \in \mathcal{G}_{x}^{x}} f(g) \varphi_{x}^{\prime}\left(u_{g}\right) d \mu(x)
$$

when $f \in C_{c}(\mathcal{G})$ and $k \in C_{c}\left(\mathcal{G}^{(0)}\right)$. It follows from this that

$$
\sum_{g \in \mathcal{G}_{x}^{x}} f(g) \varphi_{x}\left(u_{g}\right)=\sum_{g \in \mathcal{G}_{x}^{x}} f(g) \varphi_{x}^{\prime}\left(u_{g}\right)
$$

for $\mu$-almost all $x \in \mathcal{G}^{(0)}$ and all $f \in C_{c}(\mathcal{G})$. Thanks to the separability of $C^{*}(\mathcal{G})$ we conclude that $\varphi_{x}=\varphi_{x}^{\prime}$ for $\mu$-almost all $x$.

Corollary 3.3. Let $\mathcal{G}$ be a locally compact second countable Hausdorff étale groupoid and let $c: \mathcal{G} \rightarrow \mathbb{R}$ be a continuous homomorphism. Assume that the unit space $\mathcal{G}^{(0)}$ of $\mathcal{G}$ is totally disconnected and that the isotropy groups $\mathcal{G}_{x}^{x}, x \in \mathcal{G}^{(0)}$, are all amenable.

There is a bijective correspondence between the $\beta$-KMS weights for $\sigma^{c}$ on $C_{r}^{*}(\mathcal{G})$ and the pairs $\left(\mu,\left\{\varphi_{x}\right\}_{x \in \mathcal{G}^{(0)}}\right)$, where $\mu$ is a regular Borel measure on $\mathcal{G}^{(0)}$ and $\left\{\varphi_{x}\right\}_{x \in \mathcal{G}^{(0)}}$ is a $\mu$-measurable field of states $\varphi_{x}$ on $C_{r}^{*}\left(\mathcal{G}_{x}^{x}\right)$ such that

a) $\mu$ is quasi-invariant with cocycle $e^{-\beta c}$,

b) $\varphi_{x}\left(u_{g}\right)=\varphi_{r(h)}\left(u_{h g h^{-1}}\right)$ for $\mu$-almost every $x \in \mathcal{G}^{(0)}$ and all $g \in \mathcal{G}_{x}^{x}, h \in \mathcal{G}_{x}$, and

c) $\varphi_{x}\left(u_{g}\right)=0$ for $\mu$-almost every $x \in \mathcal{G}^{(0)}$ and all $g \in \mathcal{G}_{x}^{x} \backslash c^{-1}(0)$.

The $\beta$-KMS weight $\phi$ corresponding to the pair $\left(\mu,\left\{\varphi_{x}\right\}_{x \in \mathcal{G}^{(0)}}\right)$ has the properties that $C_{c}(\mathcal{G}) \subseteq \mathcal{M}_{\phi}$ and

$$
\phi(f)=\int_{\mathcal{G}^{(0)}} \sum_{g \in \mathcal{G}_{x}^{x}} f(g) \varphi_{x}\left(u_{g}\right) d \mu(x)
$$

when $f \in C_{c}(\mathcal{G})$.

Proof. It suffices to show that the assumption on the isotropy groups implies that every $\beta$-KMS weight $\phi$ on $C^{*}(\mathcal{G})$ factorises through $C_{r}^{*}(\mathcal{G})$. To this end note that it follows from Lemma 2.1 in [Th2] that for each $n \in \mathbb{N}$ there is a bounded $\beta$-KMS weight $\tilde{\phi}_{n}$ on $p_{n} C_{r}^{*}(\mathcal{G}) p_{n}$ such that $\tilde{\phi}_{n}\left(p_{n} \pi(a) p_{n}\right)=\phi\left(p_{n} a p_{n}\right)$ for all $a \in C^{*}(\mathcal{G})$ where $\pi: C^{*}(\mathcal{G}) \rightarrow C_{r}^{*}(\mathcal{G})$ is the canonical surjection. Then $\tilde{\phi}_{n}\left(p_{n} b p_{n}\right) \leq \tilde{\phi}_{n+1}\left(p_{n+1} b p_{n+1}\right)$ for all $b \geq 0$ in $C_{r}^{*}(\mathcal{G})$ and we can define a lower semi-continuous weight $\tilde{\phi}$ on $C_{r}^{*}(\mathcal{G})$ such that $\tilde{\phi}(b)=\lim _{n \rightarrow \infty} \tilde{\phi}_{n}\left(p_{n} b p_{n}\right)$. It follows that $\tilde{\phi} \circ \pi=\phi$.

It is an interesting problem if Corollary 3.3 remains true without the amenability assumption on the isotropy groups. For the proof of our main result the following suffices. 
Corollary 3.4. Let $\mathcal{G}$ be a locally compact second countable Hausdorff étale groupoid and let $c: \mathcal{G} \rightarrow \mathbb{R}$ be a continuous homomorphism. Assume that the unit space $\mathcal{G}^{(0)}$ of $\mathcal{G}$ is totally disconnected. If there is a $\beta$-KMS weight for $\sigma^{c}$ on $C_{r}^{*}(\mathcal{G})$ there is also one which is diagonal.

Proof. Let $\phi$ be a $\beta$-KMS weight for $\sigma^{c}$ on $C_{r}^{*}(\mathcal{G})$ and let $\pi: C^{*}(\mathcal{G}) \rightarrow C_{r}^{*}(\mathcal{G})$ be the canonical surjection. Then $\phi \circ \pi$ is a $\beta$-KMS weight for $\sigma^{c}$ on $C^{*}(\mathcal{G})$ and we can consider the corresponding regular Borel measure $\mu$. Since $\mu$ is quasi-invariant with cocycle $e^{-\beta c}$ it follows from Proposition 2.1 in [Th3] that $\mu$ defines a diagonal $\beta$-KMS weight by the formula (2.2).

4. Conditions on a KMS Weight that imply diagonality of the aCtion

4.1. When KMS weights factor through the conditional expectation onto an abelian subalgebra. A weight $\omega$ is faithful when $a \geq 0, \omega(a)=0 \Rightarrow a=0$.

Proposition 4.1. Let $A$ be $a C^{*}$-algebra and $\gamma$ a continuous one-parameter group of automorphisms on $A$. Let $D \subseteq A$ be an abelian $C^{*}$-subalgebra and $P: A \rightarrow D$ a conditional expectation.

Assume that $\omega$ is a faithful $\beta$-KMS weight for $\gamma, \beta \neq 0$, such that $\omega \circ P=\omega$. It follows that $\gamma_{t}(d)=d$ for all $t \in \mathbb{R}$ and all $d \in D$.

Proof. Let $f \in D, f \geq 0$. Since $\omega$ is densely defined there is a sequence $\left\{a_{n}\right\}$ of positive elements in $A$ such that $\lim _{n \rightarrow \infty} a_{n}=f$ and $\omega\left(a_{n}\right)<\infty$ for all $n$. Then $\lim _{n \rightarrow \infty} P\left(a_{n}\right)=f$ and $\omega\left(P\left(a_{n}\right)\right)=\omega\left(a_{n}\right)<\infty$. It suffices therefore to consider $f \in D, f \geq 0$ such that $\omega(f)<\infty$ and show that $\gamma_{t}(f)=f$ for all $t \in \mathbb{R}$.

We find that

$$
\omega(a f)=\omega(P(a) f)=\omega(f P(a))=\omega(f a)
$$

for all $a \in \mathcal{M}_{\omega}$. Since $\gamma_{t}\left(\mathcal{M}_{\omega}\right)=\mathcal{M}_{\omega}$ and $\omega \circ \gamma_{t}=\omega$ for all $t$, it follows from (4.1) that

$$
\omega\left(a \gamma_{t}(f)\right)=\omega\left(\gamma_{t}(f) a\right)
$$

for all $a \in \mathcal{M}_{\omega}$ and all $t \in \mathbb{R}$. For $k \in \mathbb{N}, a \in A$, set

$$
Q_{k}(a)=\sqrt{\frac{k}{\pi}} \int_{\mathbb{R}} e^{-k t^{2}} \gamma_{t}(a) d t .
$$

Note that $Q_{k}(a)$ is analytic for $\gamma$ and that $\lim _{k \rightarrow \infty} Q_{k}(a)=a$.

Observation 4.2. Assume that $a \in \mathcal{M}_{\omega}$. It follows that

$$
\sqrt{\frac{k}{\pi}} \int_{\mathbb{R}} e^{-k(t+i s)^{2}} \gamma_{t}(a) d t \in \mathcal{M}_{\omega}
$$

for all $s \in \mathbb{R}$.

Indeed,

$$
\sqrt{\frac{k}{\pi}} \int_{\mathbb{R}} e^{-k(t+i s)^{2}} \gamma_{t}(a) d t=e^{k s^{2}} \sqrt{\frac{k}{\pi}} \int_{\mathbb{R}} e^{-k t^{2}}(\cos (2 k s t)-i \sin (2 k s t)) \gamma_{t}(a) d t,
$$

so it suffices to show that

$$
x=\sqrt{\frac{k}{\pi}} \int_{\mathbb{R}} g(t) e^{-k t^{2}} \gamma_{t}(a) d t \in \mathcal{M}_{\omega}
$$


when $a \geq 0, \omega(a)<\infty$ and $g \geq 0$ is continuous and bounded. Since

$$
\sqrt{\frac{k}{\pi}} \int_{\mathbb{R}} e^{-k t^{2}} d t=1
$$

it follows that $x$ is the norm limit of a sequence $\left\{x_{n}\right\}$ of elements of the form

$$
x_{n}=\sum_{i=1}^{N} \alpha_{i} g\left(t_{i}\right) \gamma_{t_{i}}(a)
$$

where $\alpha_{i} \geq 0$ and $\sum_{i=1}^{N} \alpha_{i}=1$. Note that

$$
\omega\left(x_{n}\right)=\sum_{i=1}^{N} \alpha_{i} g\left(t_{i}\right) \omega\left(\gamma_{t_{i}}(a)\right)=\sum_{i=1}^{N} \alpha_{i} g\left(t_{i}\right) \omega(a) \leq \omega(a) \sup _{t \in \mathbb{R}} g(t) .
$$

Since $\omega$ is lower semi-continuous, it follows that $\omega(x) \leq \omega(a) \sup _{t \in \mathbb{R}} g(t)<\infty$, completing the proof of Observation 4.2 .

Since

$$
\gamma_{i s}\left(Q_{k}(a)\right)=\sqrt{\frac{k}{\pi}} \int_{\mathbb{R}} e^{-k(t-i s)^{2}} \gamma_{t}(a) d t
$$

it follows from Observation 4.2 that

$$
\gamma_{i s}\left(Q_{k}(a)\right) \in \mathcal{M}_{\omega}
$$

when $a \in \mathcal{M}_{\omega}$ and for all $s \in \mathbb{R}$. Let $a, b \in \mathcal{M}_{\omega}, t \in \mathbb{R}$. Assume that $a$ and $b$ are analytic for $\gamma$, and that $\gamma_{i \beta}(a), \gamma_{i \beta}(b) \in \mathcal{M}_{\omega}$. Then

$$
\begin{array}{lr}
\omega\left(a \gamma_{t}(f) b\right)=\omega\left(\gamma_{t}(f) b \gamma_{i \beta}(a)\right) & \text { (using Proposition 1.12 (3) in [KV]) } \\
=\omega\left(b \gamma_{i \beta}(a) \gamma_{t}(f)\right) & \text { (using (4.2)) } \\
=\omega\left(\gamma_{i \beta}(a) \gamma_{t}(f) \gamma_{i \beta}(b)\right) & \text { (using Proposition 1.12 (3) in [KV]). }
\end{array}
$$

Note that when $a, c \in \mathcal{M}_{\omega}$, the Cauchy-Schwarz inequality yields the estimate

$$
|\omega(a b c)| \leq\|b\| \omega\left(a a^{*}\right)^{\frac{1}{2}} \omega\left(c^{*} c\right)^{\frac{1}{2}}
$$

for all $b \in A$. In particular, $x \mapsto \omega(a x b)$ and $x \mapsto \omega\left(\gamma_{i \beta}(a) x \gamma_{i \beta}(b)\right)$ are both bounded functionals on $A$ and we conclude therefore from (4.4) that

$$
\omega\left(a Q_{k}(f) b\right)=\omega\left(\gamma_{i \beta}(a) Q_{k}(f) \gamma_{i \beta}(b)\right)
$$

for all $k \in \mathbb{N}$. Since

$$
\begin{aligned}
& \omega\left(\gamma_{i \beta}(a) Q_{k}(f) \gamma_{i \beta}(b)\right)=\omega \circ \gamma_{i \beta}\left(a \gamma_{-i \beta}\left(Q_{k}(f)\right) b\right) \\
& =\omega\left(a \gamma_{-i \beta}\left(Q_{k}(f)\right) b\right)
\end{aligned}
$$

it follows that

$$
\omega\left(a\left[Q_{k}(f)-\gamma_{-i \beta}\left(Q_{k}(f)\right)\right] b\right)=0 .
$$

Note now that it follows from (4.3) that $Q_{k}(f)-\gamma_{-i \beta}\left(Q_{k}(f)\right) \in \mathcal{M}_{\omega}$. Since $\mathcal{M}_{\omega}$ is a $*$-algebra we conclude that

$$
\left(Q_{k}(f)-\gamma_{-i \beta}\left(Q_{k}(f)\right)\right)^{*} \text { and }\left(Q_{k}(f)-\gamma_{-i \beta}\left(Q_{k}(f)\right)\right)^{*}\left(Q_{k}(f)-\gamma_{-i \beta}\left(Q_{k}(f)\right)\right)
$$

are also elements of $\mathcal{M}_{\omega}$. Similarly,

$$
\gamma_{i \beta}\left(\left(Q_{k}(f)-\gamma_{-i \beta}\left(Q_{k}(f)\right)\right)^{*}\right)=\gamma_{i \beta}\left(Q_{k}(f)\right)-\gamma_{2 i \beta}\left(Q_{k}(f)\right) \in \mathcal{M}_{\omega}
$$


and

$$
\begin{aligned}
& \gamma_{i \beta}\left(\left(Q_{k}(f)-\gamma_{-i \beta}\left(Q_{k}(f)\right)\right)^{*}\left(Q_{k}(f)-\gamma_{-i \beta}\left(Q_{k}(f)\right)\right)\right) \\
& =\gamma_{i \beta}\left(\left(Q_{k}(f)-\gamma_{-i \beta}\left(Q_{k}(f)\right)\right)^{*}\right) \gamma_{i \beta}\left(Q_{k}(f)-\gamma_{-i \beta}\left(Q_{k}(f)\right)\right) \in \mathcal{M}_{\omega} .
\end{aligned}
$$

We can then choose

$$
a=\left(Q_{k}(f)-\gamma_{-i \beta}\left(Q_{k}(f)\right)\right)^{*}
$$

and

$$
b=\left(Q_{k}(f)-\gamma_{-i \beta}\left(Q_{k}(f)\right)\right)^{*}\left(Q_{k}(f)-\gamma_{-i \beta}\left(Q_{k}(f)\right)\right)
$$

in (4.5) to deduce that $\omega\left(b^{2}\right)=0$. Since $\omega$ is faithful by assumption it follows that $b=0$, i.e.

$$
Q_{k}(f)=\gamma_{-i \beta}\left(Q_{k}(f)\right) .
$$

Since $Q_{k}(f)$ is analytic for $\gamma$ it follows that

$$
\gamma_{z}\left(Q_{k}(f)\right)=\gamma_{z-i \beta}\left(Q_{k}(f)\right)
$$

for all $z \in \mathbb{C}$. Set

$$
D=\sup \left\{\left\|\gamma_{i s}\left(Q_{k}(f)\right)\right\|: s \in[-|\beta|,|\beta|]\right\} .
$$

Using (4.6) it follows that $\left\|\gamma_{t+i s}\left(Q_{k}(f)\right)\right\|=\left\|\gamma_{i s}\left(Q_{k}(f)\right)\right\| \leq D$ for all $t, s \in \mathbb{R}$. Then Liouvilles theorem implies that

$$
z \mapsto \gamma_{z}\left(Q_{k}(f)\right)
$$

is constant, i.e. $\gamma_{t}\left(Q_{k}(f)\right)=Q_{k}(f)$ for all $t \in \mathbb{R}$. This conclusion holds for all $k \in \mathbb{N}$ and since $\lim _{k \rightarrow \infty} Q_{k}(f)=f$, this completes the proof.

Corollary 4.3. Let $A$ be a simple $C^{*}$-algebra and $\gamma$ a continuous one-parameter group of automorphisms on $A$. Let $D \subseteq A$ be an abelian $C^{*}$-subalgebra and $P: A \rightarrow$ $D$ a conditional expectation.

Assume that $\omega$ is a $\beta$-KMS weight for $\gamma, \beta \neq 0$, such that $\omega \circ P=\omega$. It follows that $\gamma_{t}(d)=d$ for all $t \in \mathbb{R}$ and all $d \in D$.

Proof. It suffices to show that $\omega$ is faithful. So assume that $b=b^{*} \in A$ and that $\omega\left(b^{2}\right)=0$. For a $c \in \mathcal{M}_{\omega}$ we know from the above proof that $Q_{k}(c), \gamma_{i \beta}\left(Q_{k}(c)^{*}\right) \in$ $\mathcal{M}_{\omega}$, hence by an application of the Cauchy-Schwarz inequality

$$
\left|\omega\left(Q_{k}(c)^{*} b^{2} Q_{k}(c)\right)\right|^{2}=\left|\omega\left(b^{2} Q_{k}(c) \gamma_{i \beta}\left(Q_{k}(c)^{*}\right)\right)\right|^{2} \leq 0 .
$$

Lower semi-continuity now implies that $\omega\left(c^{*} b^{2} c\right)=0$ and by using Cauchy-Schwarz again we deduce that

$$
\omega\left(\operatorname{Span} \mathcal{M}_{\omega} b^{2} \mathcal{M}_{\omega}\right)=\{0\} .
$$

Since $\mathcal{M}_{\omega}$ is dense in $A$ the closure of $\operatorname{Span} \mathcal{M}_{\omega} b^{2} \mathcal{M}_{\omega}$ is a (closed twosided) ideal in $A$. If $b \neq 0$ this ideal must be all of $A$ because we assume that $A$ is simple. But then we reach a contradiction the following way: Let $a \geq 0$. Choose a sequence $\left\{x_{n}\right\} \subseteq \operatorname{Span} \mathcal{M}_{\omega} b^{2} \mathcal{M}_{\omega}$ such that $\lim _{n \rightarrow \infty} x_{n}=\sqrt{a}$. Since $x_{n} x_{n}^{*} \in \operatorname{Span} \mathcal{M}_{\omega} b^{2} \mathcal{M}_{\omega}$ and $\lim _{n \rightarrow \infty} x_{n} x_{n}^{*}=a$, it follows from (4.7) and the lower semi-continuity of $\omega$ that $\omega(a)=0$. This is a contradiction because $\omega \neq 0$. Hence $b=0$. 


\subsection{One-parameter groups trivial on the diagonal.}

Proposition 4.4. Let $\mathcal{G}$ be a locally compact Hausdorff étale groupoid and $\alpha=$ $\left(\alpha_{t}\right)_{t \in \mathbb{R}}$ a continuous one-parameter group of automorphisms on $C_{r}^{*}(\mathcal{G})$ such that

$$
\alpha_{t}(f)=f
$$

for all $f \in C_{0}\left(\mathcal{G}^{(0)}\right)$ and all $t \in \mathbb{R}$. Assume that the elements of $\mathcal{G}^{(0)}$ with trivial isotropy group in $\mathcal{G}$ are dense in $\mathcal{G}^{(0)}$. There is a continuous homomorphism $c: \mathcal{G} \rightarrow$ $\mathbb{R}$ such that

$$
\alpha_{t}(g)(\xi)=e^{i t c(\xi)} g(\xi)
$$

for all $t \in \mathbb{R}$, all $g \in C_{c}(\mathcal{G})$ and all $\xi \in \mathcal{G}$.

Proof. We shall use the continuous linear embedding $j: C_{r}^{*}(\mathcal{G}) \rightarrow C_{0}(\mathcal{G})$ introduced by Renault in Proposition 4.2 in [Re1].

Observation 4.5. Let $f \in C_{c}(\mathcal{G})$ be supported in an open subset $U \subseteq \mathcal{G}$ such that $r: U \rightarrow \mathcal{G}^{(0)}$ is injective. Assume that $f(\xi)=0$ for some $\xi \in U$. It follows that $j\left(\alpha_{t}(f)\right)(\xi)=0$ for all $t \in \mathbb{R}$.

To prove this, let $\epsilon>0$. There is an open bisection $W$ of $\xi$ such that $W \subseteq U$ and $|f(\mu)| \leq \epsilon$ for all $\mu \in W$. Let $\varphi \in C_{c}\left(\mathcal{G}^{(0)}\right)$ be such that $0 \leq \varphi \leq 1, \operatorname{supp} \varphi \subseteq r(W)$ and $\varphi(r(\xi))=1$. By use of Proposition 4.2 in [Re1] we find that

$$
j\left(\alpha_{t}(f)\right)(\xi)=\varphi(r(\xi)) j\left(\alpha_{t}(f)\right)(\xi)=j\left(\varphi \alpha_{t}(f)\right)(\xi)=j\left(\alpha_{t}(\varphi f)\right)(\xi) .
$$

Note that $\operatorname{supp}(\varphi f) \subseteq W$ and that $\|\varphi f\|_{\infty} \leq \epsilon$. It follows that

$$
\left\|j\left(\alpha_{t}(\varphi f)\right)\right\|_{\infty} \leq\left\|\alpha_{t}(\varphi f)\right\|=\|\varphi f\|=\|\varphi f\|_{\infty} \leq \epsilon,
$$

where the last identity follows from Lemma 2.4 in [Th1]. In particular, $\left|j\left(\alpha_{t}(\varphi f)\right)(\xi)\right| \leq$ $\epsilon$, and then (4.8) shows that $\left|j\left(\alpha_{t}(f)\right)(\xi)\right| \leq \epsilon$. This proves Observation 4.5,

In the same way we obtain the following

Observation 4.6. Let $f \in C_{c}(\mathcal{G})$ be supported in an open subset $U \subseteq \mathcal{G}$ such that $s: U \rightarrow \mathcal{G}^{(0)}$ is injective. Assume that $f(\xi)=0$ for some $\xi \in U$. It follows that $j\left(\alpha_{t}(f)\right)(\xi)=0$ for all $t \in \mathbb{R}$.

Observation 4.7. Let $\xi \in \mathcal{G}$, and let $h, h^{\prime} \in C_{c}(\mathcal{G})$ be supported in (not necessarily the same) open bisections in $\mathcal{G}$. Assume that $h(\xi)=h^{\prime}(\xi)=1$. Then

$$
j\left(\alpha_{t}(h)\right)(\xi)=j\left(\alpha_{t}\left(h^{\prime}\right)\right)(\xi)
$$

for all $t \in \mathbb{R}$.

To show this, let $h \cdot h^{\prime}$ be the pointwise product of $h$ and $h^{\prime}$. It follows from Observation 4.5 that

$$
j\left(\alpha_{t}\left(h \cdot h^{\prime}-h^{\prime}\right)\right)(\xi)=j\left(\alpha_{t}\left(h \cdot h^{\prime}-h\right)\right)(\xi)=0,
$$

which yields (4.9): $j\left(\alpha_{t}(h)\right)(\xi)=j\left(\alpha_{t}\left(h \cdot h^{\prime}\right)\right)(\xi)=j\left(\alpha_{t}\left(h^{\prime}\right)\right)(\xi)$.

It follows from Observation 4.7 that we can define a map $G_{t}: \mathcal{G} \rightarrow \mathbb{C}$ such that

$$
G_{t}(\xi)=j\left(\alpha_{t}(h)\right)(\xi),
$$

where $h$ is any element of $C_{c}(\mathcal{G})$ which is supported in an open bisection and takes the value 1 at $\xi$. Note that $G_{t}$ is continuous by construction. 
Observation 4.8. For every $f \in C_{c}(\mathcal{G})$ and every $\xi \in \mathcal{G}$,

$$
j\left(\alpha_{t}(f)\right)(\xi)=G_{t}(\xi) f(\xi) .
$$

To show this, we may assume that there are open bisections $U \subseteq V$ such that $\operatorname{supp} f \subseteq U$ and $\bar{U} \subseteq V$. Assume first that $\xi \notin \bar{U}$. We must show that $j\left(\alpha_{t}(f)\right)(\xi)=$ 0 in this case. By continuity and the assumption on $\mathcal{G}$ we may assume that $s(\xi)$ has trivial isotropy. If $\mu \in U$ and $r(\mu)=r(\xi), s(\mu)=s(\xi)$, we see that

$$
r\left(\mu^{-1} \xi\right)=s(\mu)=s(\xi) \text { and } s\left(\mu^{-1} \xi\right)=s(\xi)
$$

which is impossible since $\xi \neq \mu$. It follows that we can write $f$ as a finite sum

$$
f=\sum_{i} f_{i}
$$

such that each $f_{i} \in C_{c}(U)$ is supported in an open set $W_{i} \subseteq U$ such that either $s(\xi) \notin s\left(\overline{W_{i}}\right)$ or $r(\xi) \notin r\left(\overline{W_{i}}\right)$. It follows that $j\left(\alpha_{t}\left(f_{i}\right)\right)(\xi)=0$; in the first case thanks to Observation 4.6, in the second thanks to Observation 4.5. Hence

$$
j\left(\alpha_{t}(f)\right)(\xi)=\sum_{i} j\left(\alpha_{t}\left(f_{i}\right)\right)(\xi)=0,
$$

as desired. Assume then that $\xi \in \bar{U} \subseteq V$. Choose $\epsilon>0$ such that $f(\xi)+\epsilon \neq 0$ and a function $\varphi \in C_{c}(V)$ such that $\varphi(\xi)=1$. Then

$$
j\left(\alpha_{t}(f+\epsilon \varphi)\right)(\xi)=j\left(\alpha_{t}\left(\frac{f+\epsilon \varphi}{f(\xi)+\epsilon}\right)\right)(\xi)(f(\xi)+\epsilon)=G_{t}(\xi)(f(\xi)+\epsilon) .
$$

Letting $\epsilon \rightarrow 0$ we obtain (4.10).

Note that it follows from Observation 4.8 that $\alpha_{t}\left(C_{c}(\mathcal{G})\right) \subseteq C_{c}(\mathcal{G})$, and

$$
\alpha_{t}(f)(\xi)=G_{t}(\xi) f(\xi)
$$

for all $f \in C_{c}(\mathcal{G})$ and all $\xi \in \mathcal{G}$. Since $\|f\|=\left\|\alpha_{t}(f)\right\|$ this implies that $\left|G_{t}(\xi)\right|=1$. Furthermore, if $h \in C_{c}(\mathcal{G})$ is supported in a bisection and $h(\xi)=1$, we find that

$$
\begin{aligned}
& G_{t+s}(\xi)=\alpha_{t}\left(\alpha_{s}(h)\right)(\xi) \\
& =\alpha_{s}(h)(\xi) \alpha_{t}\left(\frac{\alpha_{s}(h)}{\alpha_{s}(h)(\xi)}\right)(\xi)=\alpha_{s}(h)(\xi) G_{t}(\xi) \\
& =G_{s}(\xi) G_{t}(\xi) .
\end{aligned}
$$

Since $t \mapsto G_{t}(\xi)$ is continuous, this implies that there is a unique real-valued function $c: \mathcal{G} \rightarrow \mathbb{R}$ such that

$$
G_{t}(\xi)=e^{i t c(\xi)}
$$

To show that $c$ is a homomorphism, let $\gamma_{1}, \gamma_{2} \in \mathcal{G}$ such that $s\left(\gamma_{1}\right)=r\left(\gamma_{2}\right)$. Set $\gamma=\gamma_{1} \gamma_{2}$. Let $U$ be an open bisection containing $\gamma$ and $U_{i}$ an open bisection containing $\gamma_{i}, i=1,2$, such that $\mu_{1} \mu_{2} \in U$ when $\left(\mu_{1}, \mu_{2}\right) \in \mathcal{G}^{(2)} \cap\left(U_{1} \times U_{2}\right)$. Choose $h_{i} \in C_{c}\left(U_{i}\right)$ such that $h_{i}\left(\gamma_{i}\right)=1$. Then $h_{1} h_{2}(\gamma)=1$ and

$$
G_{t}(\gamma)=j\left(\alpha_{t}\left(h_{1} h_{2}\right)\right)(\gamma)=\alpha_{t}\left(h_{1}\right) \alpha_{t}\left(h_{2}\right)(\gamma)=\alpha_{t}\left(h_{1}\right)\left(\gamma_{1}\right) \alpha_{t}\left(h_{2}\right)\left(\gamma_{2}\right)=G_{t}\left(\gamma_{1}\right) G_{t}\left(\gamma_{2}\right) \text {. }
$$

Hence $G_{t}$ is a homomorphism as asserted. Combining (4.11) and (4.12) and taking derivatives with respect to $t$, it follows that $c$ is a homomorphism, i.e.

$$
c\left(\gamma_{1} \gamma_{2}\right)=c\left(\gamma_{1}\right)+c\left(\gamma_{2}\right)
$$

when $s\left(\gamma_{1}\right)=r\left(\gamma_{2}\right)$. 
Finally, to show that $c$ is continuous, let $\xi \in \mathcal{G}$ and $\epsilon>0$ be given. Choose open bisections $U \subseteq V$ such that $\xi \in U \subseteq \bar{U} \subseteq V$ and $h \in C_{c}(V)$ a function such that $h=1$ on $\bar{U}$. Then

$$
G_{t}(\gamma)=\alpha_{t}(h)(\gamma)
$$

for all $t \in \mathbb{R}$ and all $\gamma \in U$. Let $K \subseteq \mathbb{R}$ be a compact set. There are finitely many points $t_{i} \in K, i=1,2, \cdots, N$, such that for every $t \in K$ there is an $i$ such that

$$
\left\|\alpha_{t}(h)-\alpha_{t_{i}}(h)\right\|_{\infty}=\left\|\alpha_{t}(h)-\alpha_{t_{i}}(h)\right\| \leq \epsilon .
$$

By continuity of $\alpha_{t_{i}}(h)$ there is an open neighborhood $W \subseteq U$ of $\xi$ such that

$$
\left|\alpha_{t_{i}}(h)(\gamma)-\alpha_{t_{i}}(h)(\xi)\right| \leq \epsilon
$$

for all $\gamma \in W$ and $i=1,2, \ldots, N$. It follows that $\left|G_{t}(\gamma)-G_{t}(\xi)\right| \leq 3 \epsilon$ for all $t \in K$ and all $\gamma \in W$. By Pontryagin duality this implies that $c$ is continuous.

Theorem 4.9. Let $\mathcal{G}$ be a locally compact Hausdorff étale groupoid such that for at least one element $x \in \mathcal{G}^{(0)}$ the isotropy $\mathcal{G}_{x}^{x}$ is trivial, i.e. $\mathcal{G}_{x}^{x}=\{x\}$, and that $\mathcal{G}$ is minimal in the sense that $s\left(r^{-1}(y)\right)$ is dense in $\mathcal{G}^{(0)}$ for all $y \in \mathcal{G}^{(0)}$. Let $\alpha=\left(\alpha_{t}\right)_{t \in \mathbb{R}}$ be a continuous one-parameter group of automorphisms on $C_{r}^{*}(\mathcal{G})$ and assume that for some $\beta \in \mathbb{R} \backslash\{0\}$ there is a diagonal $\beta$-KMS weight for $\alpha$. Then $\alpha$ is diagonal, i.e. there is a continuous homomorphism $c: \mathcal{G} \rightarrow \mathbb{R}$ such that

$$
\alpha_{t}(g)(\xi)=e^{i t c(\xi)} g(\xi)
$$

for all $t \in \mathbb{R}$, all $g \in C_{c}(\mathcal{G})$ and all $\xi \in \mathcal{G}$.

Proof. Combine Corollary 4.3 and Proposition 4.4, using that in the presence of a single unit with trivial isotropy group the minimality of $\mathcal{G}$ is equivalent to the simplicity of $C_{r}^{*}(\mathcal{G})$ by Corollary 2.18 in [Th1].

We can now put the pieces together for a proof of Theorem 2.1: 1) $\Rightarrow 3$ ) follows from Proposition 4.1. That 3) is equivalent to 4) follows from a standard argument using that $\mathcal{G}^{(0)}$ is totally disconnected. The implication 3) $\Rightarrow 5$ ) follows from Proposition 4.4 and 5$) \Rightarrow 2$ ) from Corollary 3.4. This gives the equivalence of all five conditions since 2$) \Rightarrow 1$ ) is trivial.

Example 4.10. Let $\mathcal{G}=\mathbb{F}_{2}$ be the free group on two generators. Then $C_{r}^{*}\left(\mathbb{F}_{2}\right)$ is a simple $C^{*}$-algebra and $C_{0}\left(\mathcal{G}^{(0)}\right)=\mathbb{C} 1$. Let $A=A^{*} \in C_{r}^{*}\left(\mathbb{F}_{2}\right)$ and set

$$
\alpha_{t}(a)=e^{i t A} a e^{-i t A} \text {. }
$$

Note that $\alpha_{t}$ acts trivially on $C_{0}\left(\mathcal{G}^{(0)}\right)=\mathbb{C} 1$. Let $U_{x}, x \in \mathbb{F}_{2}$, be the canonical unitaries generating $C_{r}^{*}\left(\mathbb{F}_{2}\right)$. Assume that there is a homomorphism $c: \mathbb{F}_{2} \rightarrow \mathbb{R}$ such that $\alpha_{t}\left(U_{x}\right)=e^{i t c(x)} U_{x}$ for all $t, x$. By differentiation this leads to the conclusion that $A U_{x}-U_{x} A=c(x) U_{x}$ and hence that $U_{x}^{*} A U_{x}=A+c(x) 1$. The last equation implies that the spectrum $\sigma(A)$ of $A$ satisfies $\sigma(A)=\sigma(A)+c(x)$, i.e. $c(x)=0$. But then $\alpha_{t}\left(U_{x}\right)=U_{x}$ for all $t, x$, i.e. $\alpha_{t}=$ id for all $t \in \mathbb{R}$. This implies by differentiation that $A X=X A$ for all $X \in C_{r}^{*}\left(\mathbb{F}_{2}\right)$, i.e. $A$ is in the center of $C_{r}^{*}\left(\mathbb{F}_{2}\right)$. So by choosing $A \notin \mathbb{R} 1$, we have an example showing that Proposition 4.4 does not always hold when there are no units with trivial isotropy in $\mathcal{G}$. In relation to Theorem 2.1 note that there are $\beta$-KMS weights for $\alpha$ for all $\beta \in \mathbb{R}$. Indeed, when $\omega$ is the tracial state on $C_{r}^{*}\left(\mathbb{F}_{2}\right)$, the functional

$$
C_{r}^{*}\left(\mathbb{F}_{2}\right) \ni a \mapsto \omega\left(e^{-\beta A} a\right)
$$


is a bounded $\beta$-KMS weight. Since condition 3) in Theorem 2.1 holds while 5) does not, it follows that it is necessary, in Theorem 2.1, to assume the existence of a unit with trivial isotropy group.

Similarly, by considering a disjoint union $\mathbb{F}_{2} \sqcup \mathcal{H}$, where $\mathcal{H}$ is an appropriate groupoid, it is easy to obtain examples showing that the implication 4 ) $\Rightarrow 1$ ) in Theorem 2.1 fails in general if $\mathcal{G}$ is not minimal.

\section{Applichtions to graph $C^{*}$-Algebras}

In this section we apply the results obtained above to the study of KMS weights on graph $C^{*}$-algebras. For this we first show how a graph $C^{*}$-algebra can be realized as the groupoid $C^{*}$-algebra of a locally defined local homeomorphism as it was introduced by Renault in $[\operatorname{Re} 2]$. Recall that graph $C^{*}$-algebras were originally introduced for row-finite graphs in [KPRR] as the $C^{*}$-algebra of the left-shift on the space of infinite paths in the graph. We show that in general, when the graph may have infinite emitters, its $C^{*}$-algebra is still the groupoid $C^{*}$-algebra of a local homeomorphism which is generally only defined on a dense open subset of a locally compact Hausdorff space.

5.1. The Renault groupoid of a local homeomorphism. Let $X$ be a locally compact second countable Hausdorff space. Let $U \subseteq X$ be an open subset and $\varphi: U \rightarrow X$ a local homeomorphism, i.e. for every $u \in U$ there is an open subset $V \subseteq U$ such that $u \in V, \varphi(V)$ is open and $\varphi: V \rightarrow \varphi(V)$ is a homeomorphism. Set $\varphi^{0}=\operatorname{id}_{X}$ (with domain $D\left(\varphi^{0}\right)=X$ ) and for $n \geq 1$, set

$$
D\left(\varphi^{n}\right)=U \cap \varphi^{-1}(U) \cap \varphi^{-2}(U) \cap \cdots \cap \varphi^{-n+1}(U)
$$

and let $\varphi^{n}$ be the map

$$
\varphi^{n}=\varphi \circ \varphi \circ \cdots \circ \varphi: D\left(\varphi^{n}\right) \rightarrow X .
$$

Set

$$
\mathcal{G}_{\varphi}=\left\{(x, n-m, y) \in X \times \mathbb{Z} \times X: \quad x \in D\left(\varphi^{n}\right), y \in D\left(\varphi^{m}\right), \varphi^{n}(x)=\varphi^{m}(y)\right\}
$$

which is a groupoid with product $(x, k, y)(y, l, z)=(x, k+l, z)$ and inversion $(x, k, y)^{-1}=$ $(y,-k, x)$. Sets of the form

$$
\left\{(x, n-m, y): \varphi^{n}(x)=\varphi^{m}(y), x \in W, y \in V\right\}
$$

for some open subsets $W \subseteq D\left(\varphi^{n}\right), V \subseteq D\left(\varphi^{m}\right)$, constitute a basis for a topology in $\mathcal{G}_{\varphi}$ which turns it into a locally compact second countable Hausdorff étale groupoid.

Let $F: X \rightarrow \mathbb{R}$ be a function which is continuous on $U$. We can then define $c_{F}: \mathcal{G}_{\varphi} \rightarrow \mathbb{R}$ such that

$$
c_{F}(x, n-m, y)=\sum_{i=0}^{n} F\left(\varphi^{i}(x)\right)-\sum_{i=0}^{m} F\left(\varphi^{i}(y)\right) .
$$

Note that $c_{F}$ is a continuous homomorphism, and if $F^{\prime}: X \rightarrow \mathbb{R}$ is a function which agrees with $F$ on $U$, then $c_{F^{\prime}}=c_{F}$.

Proposition 5.1. Let $c: \mathcal{G}_{\varphi} \rightarrow \mathbb{R}$ be a continuous homomorphism. There is a map $F: X \rightarrow \mathbb{R}$ which is continuous on $U$ such that $c=c_{F}$. 
Proof. Define $F: X \rightarrow \mathbb{R}$ such that

$$
F(x)= \begin{cases}c(x, 1, \varphi(x)), & x \in U \\ 0, & x \notin U\end{cases}
$$

It is straightforward to verify that $F$ is continuous on $U$ and that $c=c_{F}$.

It follows that the continuous homomorphisms $\mathcal{G} \rightarrow \mathbb{R}$ are in bijective correspondence with the continuous maps $U \rightarrow \mathbb{R}$.

A point $x \in X$ is aperiodic under $\varphi$ when

$$
x \in D\left(\varphi^{n}\right) \cap D\left(\varphi^{m}\right), \varphi^{n}(x)=\varphi^{m}(x) \Rightarrow n=m .
$$

Under the identification of $X$ with the unit space of $\mathcal{G}_{\varphi}$ the aperiodic points are the elements with trivial isotropy group. We can therefore combine Proposition 5.1 with Proposition 4.4 to obtain the following.

Proposition 5.2. Let $X$ be a locally compact second countable Hausdorff space, $U \subseteq X$ an open subset and $\varphi: U \rightarrow X$ a local homeomorphism. Assume that $\alpha=\left(\alpha_{t}\right)_{t \in \mathbb{R}}$ is a continuous one-parameter group of automorphisms on $C_{r}^{*}\left(\mathcal{G}_{\varphi}\right)$ such that

$$
\alpha_{t}(f)=f
$$

for all $f \in C_{0}(X) \subseteq C_{r}^{*}\left(\mathcal{G}_{\varphi}\right)$ and all $t \in \mathbb{R}$. Assume also that the aperiodic points of $\varphi$ are dense in $X$.

There is a continuous map $F: U \rightarrow \mathbb{R}$ such that

$$
\alpha_{t}(g)(\xi)=e^{i t c_{F}(\xi)} g(\xi)
$$

for all $t \in \mathbb{R}$, all $g \in C_{c}\left(\mathcal{G}_{\varphi}\right)$ and all $\xi \in \mathcal{G}_{\varphi}$.

For $n, m \in \mathbb{N} \cup\{0\}$, set

$$
\varphi^{-m}\left(\varphi^{n}(x)\right)= \begin{cases}\emptyset & \text { when } x \notin D\left(\varphi^{n}\right) \\ \left\{y \in D\left(\varphi^{m}\right): \varphi^{m}(y)=\varphi^{n}(x)\right\} & \text { when } x \in D\left(\varphi^{n}\right) .\end{cases}
$$

We say that $\varphi$ is minimal when

$$
\bigcup_{n, m \in \mathbb{N} \cup\{0\}} \varphi^{-m}\left(\varphi^{n}(x)\right)
$$

is dense in $X$ for all $x \in X$. Note that (5.1) is the orbit of $x$ under the action of $\mathcal{G}_{\varphi}$ on its unit space. Thus $\varphi$ is minimal iff $\mathcal{G}_{\varphi}$ is.

Proposition 5.3. Let $X$ be a locally compact second countable Hausdorff space, $U \subseteq X$ an open subset and $\varphi: U \rightarrow X$ a local homeomorphism. Assume that $\varphi$ is minimal and that there is at least one aperiodic point for $\varphi$. Let $\alpha=\left(\alpha_{t}\right)_{t \in \mathbb{R}}$ be a continuous one-parameter group of automorphisms on $C_{r}^{*}\left(\mathcal{G}_{\varphi}\right)$.

If, for some $\beta \neq 0$, there is a diagonal $\beta$-KMS weight for $\alpha$, there is a continuous map $F: U \rightarrow \mathbb{R}$ such that

$$
\alpha_{t}(g)(\xi)=e^{i t c_{F}(\xi)} g(\xi)
$$

for all $t \in \mathbb{R}$, all $g \in C_{c}\left(\mathcal{G}_{\varphi}\right)$ and all $\xi \in \mathcal{G}_{\varphi}$.

Proof. In view of Corollary 4.3 and Proposition 5.2 it suffices to observe that $C_{r}^{*}\left(\mathcal{G}_{\varphi}\right)$ is simple under the present assumptions, cf. Proposition 2.5 in [Re2]. 
5.2. A local homeomorphism from an infinite graph. Let $G$ be a directed graph with vertexes $V$ and edges $E$. We assume that $G$ is countable in the sense that $V$ and $E$ are both countable sets. We let $r$ and $s$ denote the maps $r: E \rightarrow V$ and $s: E \rightarrow V$ which associate to an edge $e \in E$ its target vertex $r(e)$ and source vertex $s(e)$, respectively. A vertex $v$ is an infinite emitter when $s^{-1}(v)$ contains infinitely many edges and a sink when $s^{-1}(v)$ is empty. The union of sinks and infinite emitters constitute a set which will be denoted by $V_{\infty}$. The graph $C^{*}$-algebra $C^{*}(G)$ is by definition the universal $C^{*}$-algebra generated by a collection $S_{e}, e \in E$, of partial isometries and a collection $P_{v}, v \in V$, of mutually orthogonal projections subject to the conditions that

1) $S_{e}^{*} S_{e}=P_{r(e)}, \forall e \in E$,

2) $\sum_{e \in F} S_{e} S_{e}^{*} \leq P_{v}$ for every finite subset $F \subseteq s^{-1}(v)$ and all $v \in V$, and

3) $P_{v}=\sum_{e \in s^{-1}(v)} S_{e} S_{e}^{*}, \forall v \in V \backslash V_{\infty}$.

Let $P_{f}(G)$ and $P(G)$ denote the set of finite and infinite paths in $G$, respectively. The range and source maps, $r$ and $s$, extend in the natural way to $P_{f}(G)$; the source map also to $P(G)$. Set $\Omega_{G}=P(G) \cup Q(G)$, where

$$
Q(G)=\left\{p \in P_{f}(G): r(p) \in V_{\infty}\right\}
$$

is the set of finite paths that terminate at a vertex in $V_{\infty}$. In particular, $V_{\infty} \subseteq Q(G)$ because vertexes are considered to be finite paths of length 0 . For any $p \in P_{f}(G)$, let $|p|$ denote the length of $p$. When $|p| \geq 1$, set

$$
Z(p)=\left\{q \in \Omega_{G}:|q| \geq|p|, q_{i}=p_{i}, i=1,2, \cdots,|p|\right\},
$$

and

$$
Z(v)=\left\{q \in \Omega_{G}: s(q)=v\right\}
$$

when $v \in V$. When $\nu \in P_{f}(G)$ and $F$ is a finite subset of $P_{f}(G)$, set

$$
Z_{F}(\nu)=Z(\nu) \backslash\left(\bigcup_{\mu \in F} Z(\mu)\right) .
$$

The sets $Z_{F}(\nu)$ form a basis of compact and open subsets for a locally compact Hausdorff topology on $\Omega_{G}$. When $\mu \in P_{f}(G)$ and $x \in \Omega_{G}$, we can define the concatenation $\mu x \in \Omega_{G}$ in the obvious way when $r(\mu)=s(x)$. The groupoid $\mathcal{G}_{G}$ consists of the elements in $\Omega_{G} \times \mathbb{Z} \times \Omega_{G}$ of the form

$$
\left(\mu x,|\mu|-\left|\mu^{\prime}\right|, \mu^{\prime} x\right)
$$

for some $x \in \Omega_{G}$ and some $\mu, \mu^{\prime} \in P_{f}(G)$. The product in $\mathcal{G}_{G}$ is defined by

$$
\left(\mu x,|\mu|-\left|\mu^{\prime}\right|, \mu^{\prime} x\right)\left(\nu y,|\nu|-\left|\nu^{\prime}\right|, \nu^{\prime} y\right)=\left(\mu x,|\mu|+|\nu|-\left|\mu^{\prime}\right|-\left|\nu^{\prime}\right|, \nu^{\prime} y\right),
$$

when $\mu^{\prime} x=\nu y$, and the involution by $\left(\mu x,|\mu|-\left|\mu^{\prime}\right|, \mu^{\prime} x\right)^{-1}=\left(\mu^{\prime} x,\left|\mu^{\prime}\right|-|\mu|, \mu x\right)$. To describe the topology on $\mathcal{G}_{G}$, let $Z_{F}(\mu)$ and $Z_{F^{\prime}}\left(\mu^{\prime}\right)$ be two sets of the form (5.3) with $r(\mu)=r\left(\mu^{\prime}\right)$. The topology we shall consider has as a basis the sets of the form

$$
\left\{\left(\mu x,|\mu|-\left|\mu^{\prime}\right|, \mu^{\prime} x\right): \mu x \in Z_{F}(\mu), \mu^{\prime} x \in Z_{F^{\prime}}\left(\mu^{\prime}\right)\right\} .
$$

With this topology $\mathcal{G}_{G}$ becomes an étale locally compact second countable Hausdorff groupoid and we can consider the reduced $C^{*}$-algebra $C_{r}^{*}\left(\mathcal{G}_{G}\right)$ as in [Re1]. As shown by Paterson in $\left[\mathrm{Pa}\right.$ there is an isomorphism $C^{*}(G) \rightarrow C_{r}^{*}\left(\mathcal{G}_{G}\right)$ which sends $S_{e}$ to $1_{e}$, where $1_{e}$ is the characteristic function of the compact and open set

$$
\left\{(e x, 1, r(e) x): x \in \Omega_{G}\right\} \subseteq \mathcal{G}_{G},
$$


and $P_{v}$ to $1_{v}$, where $1_{v}$ is the characteristic function of the compact and open set

$$
\left\{(v x, 0, v x): x \in \Omega_{G}\right\} \subseteq \mathcal{G}_{G} .
$$

In the following we use the identification $C^{*}(G)=C_{r}^{*}\left(\mathcal{G}_{G}\right)$ and identify $\Omega_{G}$ with the unit space of $\mathcal{G}_{G}$ via the embedding $\Omega_{G} \ni x \mapsto(x, 0, x)$.

Note that $\Omega_{G} \backslash V_{\infty}$ is an open subset of $\Omega_{G}$ and that we can define a local homeomorphism

$$
\sigma: \Omega_{G} \backslash V_{\infty} \rightarrow \Omega_{G}
$$

such that $\sigma$ is the usual left shift on $P(G)$, defined such that $\sigma(x)_{i}=x_{i+1}$, while $\sigma\left(e_{1} e_{2} \cdots e_{n}\right)$ is defined as follows when $e_{1} e_{2} \cdots e_{n} \in Q(G)$ :

$$
\sigma\left(e_{1} e_{2} \cdots e_{n}\right)= \begin{cases}e_{2} e_{3} \cdots e_{n}, & \text { when } n \geq 2 \\ r\left(e_{1}\right), & \text { when } n=1 .\end{cases}
$$

It is straightforward to check that there is an identification

$$
\mathcal{G}_{G}=\mathcal{G}_{\sigma},
$$

as topological groupoids. In particular, it follows that any continuous function $F: \Omega_{G} \backslash V_{\infty} \rightarrow \mathbb{R}$ defines a continuous homomorphism $c_{F}: \mathcal{G}_{G} \rightarrow \mathbb{R}$ such that

$$
c_{F}\left(\mu x,|\mu|-\left|\mu^{\prime}\right|, \mu^{\prime} x\right)=\sum_{n=0}^{|\mu|} F\left(\sigma^{n}(\mu x)\right)-\sum_{n=0}^{\left|\mu^{\prime}\right|} F\left(\sigma^{n}\left(\mu^{\prime} x\right)\right) .
$$

To simplify notation the one-parameter group $\sigma^{c_{F}}$ defined from $c_{F}$ will be denoted by $\sigma^{F}$. It follows from Proposition 5.1 that every continuous homomorphism $\mathcal{G}_{G} \rightarrow \mathbb{R}$ arises from a continuous function $F: \Omega_{G} \backslash V_{\infty} \rightarrow \mathbb{R}$ as above. We can therefore formulate Corollary 3.3 in the following way for graph $C^{*}$-algebras.

Theorem 5.4. Let $F: \Omega_{G} \backslash V_{\infty} \rightarrow \mathbb{R}$ be a continuous function. There is a bijective correspondence between the $\beta$-KMS weights for $\sigma^{F}$ on $C^{*}(G)$ and the pairs $\left(\mu,\left\{\varphi_{x}\right\}_{x \in \Omega_{G}}\right)$, where $\mu$ is a regular Borel measure on $\Omega_{G}$ and $\left\{\varphi_{x}\right\}_{x \in \Omega_{G}}$ is a $\mu$ measurable field of states $\varphi_{x}$ on $C_{r}^{*}\left(\left(\mathcal{G}_{G}\right)_{x}^{x}\right)$ such that

a) $\mu$ is $e^{\beta F}$-conformal for $\sigma$,

b) $\varphi_{x}\left(u_{g}\right)=\varphi_{r(h)}\left(u_{h g h^{-1}}\right)$ for $\mu$-almost every $x \in \Omega_{G}$ and all $g \in\left(\mathcal{G}_{G}\right)_{x}^{x}, h \in$ $\left(\mathcal{G}_{G}\right)_{x}$, and

c) $\varphi_{x}\left(u_{g}\right)=0$ for $\mu$-almost every $x \in \Omega_{G}$ and all $g \in\left(\mathcal{G}_{G}\right)_{x}^{x} \backslash c_{F}^{-1}(0)$.

The $\beta$-KMS weight $\phi$ corresponding to the pair $\left(\mu,\left\{\varphi_{x}\right\}_{x \in \Omega_{G}}\right)$ has the properties that $C_{c}\left(\mathcal{G}_{G}\right) \subseteq \mathcal{M}_{\phi}$ and

$$
\phi(f)=\int_{\Omega_{G}} \sum_{g \in\left(\mathcal{G}_{G}\right)_{x}^{x}} f(g) \varphi_{x}\left(u_{g}\right) d \mu(x)
$$

when $f \in C_{c}\left(\mathcal{G}_{G}\right)$.

Similarly, for graph $C^{*}$-algebras our main result takes the following form.

Theorem 5.5. Let $G$ be a countable directed graph such that $C^{*}(G)$ is simple. Let $\alpha=\left(\alpha_{t}\right)_{t \in \mathbb{R}}$ be a continuous one-parameter group of automorphisms on $C^{*}(G)$ and assume that for some $\beta_{0} \neq 0$ there is a $\beta_{0}$-KMS weight for $\alpha$.

The following are equivalent:

1) There is $a \beta_{1} \neq 0$ and a diagonal $\beta_{1}-K M S$ weight for $\alpha$. 
2) Whenever $\beta \neq 0$ and there is a $\beta$-KMS weight for $\alpha$, there is also a diagonal $\beta$-KMS weight for $\alpha$.

3) $\alpha_{t}(f)=f$ for all $t \in \mathbb{R}$ and all $f \in C_{0}\left(\Omega_{G}\right)$.

4) There is a continuous function $F: \Omega_{G} \backslash V_{\infty} \rightarrow \mathbb{R}$ such that $\alpha=\sigma^{F}$.

It follows from Theorem 5.4 (and Proposition 5.1) that all KMS weights for a diagonal action on the $C^{*}$-algebra of a graph without loops are diagonal. This is not true in general; not even for finite strongly connected graphs as shown in [Th4]. However, we can now show that it holds for strongly connected graphs when the function $F$ has bounded local variation in the a sense we now make precise.

Let $v$ be a vertex in $G$ and set

$$
\operatorname{Var}_{n, v}(F)=\sup _{x, y}\left|\sum_{j=0}^{n-1} F\left(\sigma^{j}(x)\right)-\sum_{j=0}^{n-1} F\left(\sigma^{j}(y)\right)\right|
$$

where we take the supremum over all pairs $x, y \in P(G)$ with the property that $x_{i}=y_{i}, i=1,2, \cdots, n$, and $s\left(x_{1}\right)=s\left(y_{1}\right)=v$. The following condition (5.6) should be compared with Bowen's condition used by Walters, cf. [W].

Proposition 5.6. Let $G$ be a countable directed graph such that $C^{*}(G)$ is simple and let $F: \Omega_{G} \backslash V_{\infty} \rightarrow \mathbb{R}$ be a continuous function such that for some vertex $v$,

$$
\sup _{n} \operatorname{Var}_{n, v}(F)<\infty \text {. }
$$

Then every KMS weight for $\sigma^{F}$ is diagonal.

Proof. The assumption that $C^{*}(G)$ is simple means that $G$ is cofinal in the sense used (e.g.) in [Th3] and that every minimal loop in $G$ has an exit, cf. [Sz]. It is easily seen that the set of vertexes $v$ for which (5.6) holds is both hereditary and saturated. Under the present assumptions it will therefore hold for all $v$. Consider a $\beta$-KMS weight $\phi$ and the pair $\left(\mu,\left\{\varphi_{x}\right\}_{x \in \Omega_{G}}\right)$ associated to it by Theorem 5.4. It suffices to show that the elements $x \in \Omega_{G}$ for which the isotropy group $\left(\mathcal{G}_{G}\right)_{x}^{x}$ is non-trivial is a null set with respect to $\mu$. The isotropy group of a point $x \in \Omega_{G}$ is non-trivial if and only if $x$ is an infinite pre-periodic path in $G$, and there are at most countably many such points. It suffices therefore to show that $\mu(\{x\})=0$ for any infinite pre-periodic path $x$. There is an $m \in \mathbb{N}$ such that $x_{0}=\sigma^{m}(x)$ is periodic. It follows from (3.2) that

$$
\mu(\{x\})=e^{-\beta \sum_{j=0}^{m-1} F\left(\sigma^{j}(x)\right)} \mu\left(\left\{x_{0}\right\}\right),
$$

so it suffices to show that $\mu\left(\left\{x_{0}\right\}\right)=0$. Since $x_{0}$ is periodic there is a finite loop $\delta$ in $G$ such that $x_{0}=\delta^{\infty}$, and since $G$ is cofinal and every loop in $G$ has an exit there is also a finite loop $\delta^{\prime}$ in $G$ such that $\delta^{\prime} \nsubseteq x_{0}$ and $s\left(\delta^{\prime}\right)=s(\delta)$. By prolonging $\delta$ and $\delta^{\prime}$ if necessary we may assume that the length of $\delta$ and $\delta^{\prime}$ are the same, say $p$. For each $k \in \mathbb{N}$ set

$$
y_{k}=\delta^{k} \delta^{\prime} x_{0} .
$$

Since $x_{0}$ is $p$-periodic it follows from (3.2) that

$$
\mu\left(\left\{x_{0}\right\}\right)=e^{-\beta \sum_{j=0}^{k p-1} F\left(\sigma^{j}\left(x_{0}\right)\right)} \mu\left(\left\{x_{0}\right\}\right),
$$

for all $k \in \mathbb{N}$, and the desired conclusion follows if $-\beta \sum_{j=0}^{k p-1} F\left(\sigma^{j}\left(x_{0}\right)\right)$ is not zero for some $k$. Consider therefore now the case where $-\beta \sum_{j=0}^{k p-1} F\left(\sigma^{j}\left(x_{0}\right)\right)=0$ for all 
$k \in \mathbb{N}$. Since (5.6) holds we find then that

$$
\left|\beta \sum_{j=0}^{k p-1} F\left(\sigma^{j}\left(y_{k}\right)\right)\right|=\left|\beta \sum_{j=0}^{k p-1} F\left(\sigma^{j}\left(y_{k}\right)\right)-\beta \sum_{j=0}^{k p-1} F\left(\sigma^{j}\left(x_{0}\right)\right)\right| \leq|\beta| K
$$

for all $k$, where $K=\sup _{n} \operatorname{Var}_{n, v}(F)$ and $v=s(\delta)$ is the source of $\delta$. Now apply (3.2) again to find that

$$
\mu\left(\left\{y_{k}\right\}\right)=e^{-\beta \sum_{j=0}^{(k+1) p-1} F\left(\sigma^{j}\left(y_{k}\right)\right)} \mu\left(\left\{x_{0}\right\}\right) .
$$

Inserting (5.7) this leads to the conclusion that

$$
\mu\left(\left\{y_{k}\right\}\right) \geq e^{-|\beta| K} e^{-\beta \sum_{j=0}^{p-1} F\left(\sigma^{j}(z)\right)} \mu\left(\left\{x_{0}\right\}\right),
$$

where $z=\delta^{\prime} x_{0}=\sigma^{k p}\left(y_{k}\right)$. Since

$$
\sum_{k=1}^{\infty} \mu\left(\left\{y_{k}\right\}\right) \leq \mu(Z(v))<\infty
$$

we conclude that $\mu\left(\left\{x_{0}\right\}\right)=0$, as desired.

It follows from Proposition 5.6 that a generalized gauge action on a graph $C^{*}$ algebra, considered for example in [Th3], where $F$ only depends on the first edge only has gauge-invariant KMS weights, at least as long as the algebra is simple.

Remark 5.7. It should be emphasized that the conclusion in Proposition 5.6 does not hold without some condition on $F$. To see this observe that the example presented in [Th4] shows that already for the canonical finite graph $G$ for which $C^{*}(G)$ is a copy of the Cuntz algebra $\mathrm{O}_{2}$, namely the graph consisting of one vertex and two arrows, there are continuous non-negative functions $F: \Omega_{G} \rightarrow \mathbb{R}$ such that $\sigma^{F}$ admits nondiagonal KMS states. In the example from [Th4 there is at least a single extremal KMS state which is diagonal, namely the extremal KMS-state corresponding to the lowest inverse temperature $\beta_{0}$. Here we want to indicate how to modify the example in Th4 to get an example where no extremal KMS state is diagonal. The basis for this is a sequence $\left\{b_{n}\right\}_{n=1}^{\infty}$ of positive numbers with the following properties:

a) $b_{n} \geq b_{n+1} \forall n$

b) $\lim _{n \rightarrow \infty} \frac{b_{n+1}}{b_{n}}=1$,

c) $\sum_{n=1}^{\infty} b_{n}<1$, and

d) $\sum_{n=1}^{\infty} b_{n}^{s}=\infty$ for all $s<1$.

We leave the reader to verify the existence of such a sequence. Set $a_{1}=-\log b_{1}$ and $a_{k}=\log b_{k-1}-\log b_{k}, k \geq 2$, and identify the infinite path space $\Omega_{G}$ with $\{0,1\}^{\mathbb{N}}$ by labelling the two arrows in $G$ by 0 and 1 . Define then $T:\{0,1\}^{\mathbb{N}} \rightarrow \mathbb{R}$ such that $T\left(\left(x_{i}\right)_{i=1}^{\infty}\right)=a_{k}$ where $k=\min \left\{i: x_{i}=0\right\}$ when $\left(x_{i}\right)_{i=1}^{\infty} \neq 1^{\infty}$, and set $T\left(1^{\infty}\right)=0$. (As in [Th4] $1^{\infty}$ is the infinite string of 1 's.) This is a continuous non-negative function. By using Theorem 2.2 in Th4 and arguing exactly as in Section 3 of [Th4], but with the sequence $\left\{n^{-1}\right\}$ replaced by $\left\{a_{n}\right\}$, it follows that there are $\beta$-KMS states for $\sigma^{T}$ if and only if $\beta \geq 1$, and for each $\beta \geq 1$ the extremal KMS states are parametrised by the circle, and none are diagonal. As guaranteed by Theorem 5.5 there are for each $\beta \geq 1$ also one which is diagonal. As explained in [Th4 it arises by integrating the extremal ones with respect to Lebesgue measure on the circle. 


\section{REFERENCES}

[BR] O. Bratteli and D.W. Robinson, Operator Algebras and Quantum Statistical Mechanics $I+I I$, Texts and Monographs in Physics, Springer Verlag, New York, Heidelberg, Berlin, 1979 and 1981.

[C] F.Combes, Poids associé à une algèbre hilbertienne à gauche, Compos.Math. 23 (1971), 49-77.

[KPRR] A. Kumjian, D. Pask, I. Raeburn and J. Renault, Graphs, Groupoids, and CuntzKrieger algebras, J. Func. Analysis 144 (1997), 505-541.

[Ku] J. Kustermans, KMS-weights on $C^{*}$-algebras, arXiv: 9704008v1.

[KV] J. Kustermans and S. Vaes, Locally compact quantum groups, Ann. Scient. Éc. Norm. Sup. 33, 2000, 837-934.

[N] S. Neshveyev, KMS states on the $C^{*}$-algebras of non-principal groupoids, J. Operator Theory 70 (2013), 513-530.

[Pa] A.L.T. Paterson, Graph inverse semigroups, groupoids and their $C^{*}$-algebras, J. Operator Theory 48 (2002), 645-662.

[Re1] J. Renault, A Groupoid Approach to $C^{*}$-algebras, LNM 793, Springer Verlag, Berlin, Heidelberg, New York, 1980.

[Re2] J. Renault, Cuntz-like algebras. Operator theoretical methods (Timisoara, 1998), 371286, Theta Found., Bucharest, 2000.

[Sz] W. Szymanski, Simplicity of Cuntz-Krieger algebras of infinite matrices, Pac. J. Math. 122 (2001), 249-256.

[Th1] K. Thomsen, Semi-étale groupoids and applications, Annales de l'Institute Fourier 60 (2010), 759-800.

[Th2] K. Thomsen, KMS-states and conformal measures, Comm. Math. Phys. 316 (2012), 615-640. DOI: 10.1007/s00220-012-1591-z

[Th3] K. Thomsen, KMS weights on groupoid and graph $C^{*}$-algebras, J. Func. Analysis 266, 2959-2988.

[Th4] K. Thomsen, Phase transition in $\mathrm{O}_{2}$, arXiv:1510.07513

[W] P. Walters, Convergence of the Ruelle operator for a function satisfying Bowen's condition, Trans. Amer. Math. Soc.353 (2001), 327-347.

E-mail address: matkt@math.au.dk johannes@math.au.dk

Department of Mathematics, Aarhus University, Ny Munkegade 118, 8000 Aarhus C, Denmark 